\title{
Online-Prüfung mit OPAL, ONYX und MAXIMA Chancen und Grenzen
}

\author{
E. Dohmen, A. Lange, B. Kraus, S. Sturm, S. Odenbach \\ Professur für Magnetofluiddynamik, Mess- und Automatisierungstechnik, Institut für Mechatronischen Maschinenbau,
} Fakultät Maschinenwesen, TU Dresden

\begin{abstract}
Der Transfer klassischer Präsenzklausuren in digitale Formate ist perspektivisch wohl unumgänglich, um als Teil einer zeitgemäßen und qualitativ hochwertigen Ausbildung auch zielorientierte sowie anspruchsvolle Leistungsüberprüfungen durchzuführen. Im Rahmen dieser Veröffentlichung skizzieren wir an konkreten Beispielen die Chancen und Grenzen der Testplattform ONYX in Zusammenspiel mit dem Computeralgebrasystem MAXIMA unter Nutzung des Lernmanagementsystems OPAL bei der Umsetzung zweier Online-Prüfungen mit bis zu 500 Studierenden. Interessant sind dabei insbesondere die beobachteten Verlagerungen der Arbeitsanteile hinsichtlich Konzeption, Klausurbetreuung und Korrektur, die wir vorstellen und deren Auswirkungen wir diskutieren.
\end{abstract}

The transfer of classical classroom examinations into digital formats is probably inevitable in the future in order to carry out goal-oriented and demanding performance examinations as part of a modern and high-quality education. In this publication, we outline the opportunities and limitations of the ONYX testing platform in conjunction with the computer algebra system MAXIMA using the learning management system OPAL in the implementation of two online examinations with up to 500 students. Of particular interest are the observed shifts in the proportion of work with regard to conception, examination supervision and correction, which we present and whose effects we discuss.

*Corresponding author: eike.dohmen@tu-dresden.de 


\section{Einleitung}

Im Sommersemester 2020 sowie im folgenden Wintersemester wurden an der Professur für Magnetofluiddynamik, Mess- und Automatisierungstechnik im Rahmen der Lehrveranstaltung "Mess- und Automatisierungstechnik" sowohl semesterbegleitende Lernstand-Selbstevaluationen für die Studierenden als auch die Semesterabschlussprüfungen erfolgreich digital umgesetzt.

Die Vorlesungsreihe "Mess- und Automatisierungstechnik" ist auf Sommer- und Wintersemester aufgeteilt und wird mit jeweils einer Prüfung abgeschlossen. Beide Prüfungen waren dabei bisher in einen Grundlagenteil mit 10 Einzelfragen sowie einen Rechenteil mit 4 komplexen Rechenaufgaben untergliedert. Die Teilnehmendenzahlen sinken erfahrungsgemäß vom ersten zum Folgesemester bei dieser Veranstaltung von über 500 Studierenden auf etwa 400. Unabhängig von der Konzeption der Prüfungen benötigte die Korrektur in jedem Semester für den gesamten Fragenteil ca. 1 Personenmonat und für jede der Rechenaufgaben ca. 0,5 Personenmonate. Pro Jahr ergaben sich für die Prüfungskorrektur damit also in etwa 6 Personenmonate Personalaufwand.

Basierend auf den positiven Rückmeldungen der vergangenen Jahre zu unterstützenden asynchronen und individualisierbaren Lernangeboten wie Vorlesungsaufzeichnungen, Skripten, Literatur und Feedbackkanälen wurde bereits 2019 mit der Planung einer ergänzenden Online-Probeklausur im Lehrmanagementsystems (LMS) OPAL [1] begonnen, um einerseits die Vor- und Nachteile einer Online-Prüfung zu analysieren und andererseits den Studierenden eine umfassende asynchrone Möglichkeit zur individuellen Wissensüberprüfung sowie zur Prüfungsvorbereitung bereit zu stellen.

Das verwendete LMS OPAL sowie die darin integrierte Test-Suite ONYX [2] werden vom ELearning Dienstleister BPS Bildungsportal Sachsen GmbH (BPS) technisch entwickelt. „Die BPS Bildungsportal Sachsen GmbH wurde Ende 2004 von 11 sächsischen Hochschulen gegründet, um diese bei der Einführung und nachhaltigen Nutzung neuer Medien in der akademischen Aus- und Weiterbildung zu unterstützen. Ihre Kernaufgabe liegt in der bestmöglichen Beförderung des breiten Einsatzes internetgestützter Lehr-/Lernszenarien in den Hochschulen Sachsens sowie diesen verbundenen Einrichtungen" [3]. Der Arbeitskreis "E-Learning der Landesrektorenkonferenz Sachsen "unterstützt die Hochschulen bei der Fortführung des mit der Initiative "Bildungsportal Sachsen" erfolgreich eingeschlagenen Weges, ihre Potenziale auf dem Gebiet des E-Learning zu bündeln und gemeinsam zu entwickeln [...." [4]. In ONYX ist das quelloffene Computeralgebrasystem MAXIMA eingebunden.

Mit Beginn der Corona Pandemie im Frühjahr 2020 wandelte sich die als Ergänzungsangebot gedachte Online-Probeklausur in eine alternativlose Notwendigkeit für eine rein digitale Gewährleistung der Lehre. Im Rahmen dieser Veröffentlichung möchten wir das Potential von mit OPAL, ONYX und MAXIMA umgesetzten Online-Prüfungen kritisch betrachten. Hierfür geben wir Einblicke in unsere Vorgehensweise, stellen ausgewählte Beispiele unserer Umsetzungen vor, präsentieren aufgetretene Probleme, Workarounds sowie Lösungen und schätzen den Zeitaufwand ab, um mit unseren Erfahrungen schließlich eine Diskussion zu Chancen und Grenzen von OnlineTests im Vergleich zu klassischen Präsenzumsetzungen anzustoßen. Daraus leiten sich auch weitergehende Fragestellungen ab: Welche Treffsicherheit wird in Bezug auf die übergeordneten Lehr-/Lernziele erreicht? Welche Komplexität kann abgebildet werden? Wie aufwändig ist eine Online-Prüfung?

Darüber hinaus werden wir auf die eingesetzte Kommunikationsplattform Element [5] (auf Basis des quelloffenen Matrix-Protokolls) eingehen, mit der die begleitende Kommunikation erfolgreich umgesetzt wurde.

In dieser Publikation werden wir chronologisch von der Prüfungskonzeption bis zur Prüfungsauswertung die oben genannten Punkte adressieren und dann ein Fazit formulieren.

\section{Konzeptentwicklung}

Zentrale Anforderung bei der Umsetzung eines Online-Prüfungskonzept auf Basis eines bestehenden, klassischen Präsenzprüfungs- 
konzeptes ist einerseits die präzise Neudefinition von Lernzielen, ggf. durch Extraktion aus bestehenden Prüfungen, und andererseits die Ableitung neuer, auf die Möglichkeiten von OPAL, ONYX und MAXIMA angepasster, umsetzungsfähiger Aufgaben unter Fokussierung dieser Lernziele. Hierbei scheint den Autoren neben der Kenntnis von möglichen Aufgabentypen (bspw. in der Testumgebung ONYX) für Prüfungen in MINT-Fächern und einem fachlichen Hintergrundwissen, vor allem ein profundes technisches Vorwissen (im Sinne von Programmierkenntnissen) zur Parametrierung von ONYX-Aufgaben ${ }^{1}$ mittels Variablen und zur Umsetzung mathematischer Fragestellungen mit dem Computeralgebrasystem MAXIMA essentiell.

Leider fehlte für die Konzepterstellung im Sommersemester 2020 an der TU Dresden noch eine zentrale Anlaufstelle, die neben organisatorischen Fragen auch didaktische, rechtliche und technische Fragen klären konnte, bei der Erstellung der Prüfungen aktiv begleitete und/oder entsprechende Kontakte vermittelte.

Mit hohem Engagement stand im folgenden Wintersemester das Team des „Zentrum für interdisziplinäre Lernen und Lehren“ (ZiLL) [6] als zentrale Anlaufstelle für eben diese Fragen zur Verfügung und unterstützte sogar begleitend bei der Erstellung von Tests. Zudem wurde an der Fakultät Maschinenwesen die "Task Force Digitale Prüfung" aus freiwilligen Expert:innen zusammengestellt, die mit umfangreichen Erfahrungen zu ingenieurspezifischen Prüfungslösungen Kolleg:innen beraten konnten.

\section{Autor:innen}

Im Zuge der Konzeptentwicklung ergibt sich auch die Frage des Autor:innenkreises zur Generierung von entsprechenden Prüfungen, denn fachliches Vorwissen gepaart mit dem notwendigen spezialisierten Vorwissen hat im besten Fall - nur ein sehr eingeschränkter Personenkreis. Uns unterstützte ein fachfremder eScout (eine Studentin mit Vorerfahrung

\footnotetext{
${ }^{1}$ Fett kursiv hervorgehobene Begriffe kennzeichnen feste Bezeichnungen oder Fachtermini aus OPAL oder ONYX. Die Markierung dient der Abgrenzung zu Begriffen aus dem allgemeinen Sprachgebrauch. Beispiel: Es wurde
}

mit dem verwendeten LMS OPAL) sehr erfolgreich, allerdings mussten ihre und unsere Erwartungen angepasst werden, da ohne entsprechendes Fachwissen natürlich keine ONYX-Aufgaben selbstständig generiert und nur sehr eingeschränkt adaptiert werden können. Dies führt dazu, dass sich die Aufgaben des eScouts auf Zuarbeiten in den Bereichen Abbildungserstellung und Einpflegen von fertig vorbereiteten Aufgaben in ONYX auf Basis detaillierter Handlungsanweisungen beschränkten.

eScouts sind daher nach den konkreten Anforderungen und ihren Befähigungen auszuwählen, wobei eine Kombination sehr verschiedener Qualifikationen (bspw. Psychologie und Physik) besonders hilfreich wäre, aber real wohl nur durch interdisziplinäre eScout-Teams abgebildet werden könnte.

War die zentrale Hürde im Sommersemester 2020 noch die schnelle Umsetzung eines Online-Prüfungskonzept aus dem bestehenden Präsenzkonzept und die damit verbundene Einarbeitung sowie Befähigung von (Mit-)Autor:innen, konnte im Wintersemester auf der erarbeiteten Basis mit sehr guten technischen Kenntnissen, bspw. bei Aufgaben-Parametrierungen, Variablendefinitionen, Zufallswerten oder MAXIMA-Funktionen, aufgebaut werden.

Im Wintersemester 2020/2021 stand uns die tatkräftige Unterstützung eines eScouts durch das Medienzentrum der TU Dresden leider nicht mehr zur Verfügung, da man möglichst viele Lehrstühle mit dem eScout-Programm erreichen wollte. In Anbetracht der vermittelten fachlichen und technischen Befähigung wurde dies von uns als sehr ungünstige Konzeption im eScout Projekt wahrgenommen, da der effektive Nutzen für unseren Lehrstuhl in Summe sehr gering war. Aus unserer Sicht sind eScouts sehr effektive Keimzellen zur personellen Unterstützung sowie Streuung von Wissen zur Umsetzung von Online-Inhalten. Wesentlich hierfür sind eine gute bis sehr gute Befähigung von Studierenden zu eScouts und aufgrund der Einarbeitungsdauer längerfris-

eine komplexe Aufgabe als ONYX-Test umgesetzt und darin jede Teilaufgabe (dieser komplexen Aufgabe) als ONYX-Aufgabe. 
tige Verträge der eScouts zur Sicherstellung eines Wissenstransfers sowie eines echten Nutzens für die Bildungseinrichtung. Auch eine Befähigung lehrstuhleigener studentischer Hilfskräfte wäre denkbar.

\section{Klausurerstellung}

Während der Klausurerstellung sind sowohl in MAXIMA als auch in ONYX und OPAL Besonderheiten und Bugs zum Vorschein gekommen, die wir im Sinne eines Wissenstransfers nun gemeinsam mit unseren Lösungen oder Workarounds vorstellen möchten.

Bei der Nutzung des Algebrasystems MAXIMA sind einige Besonderheiten bei der Nutzung zu beachten. MAXIMA berücksichtigt bei logischen Ausdrücken beispielsweise das Kommutativgesetz nicht, d. h. "a AND b" ist nicht zwangsläufig gleich „b AND a“ und auch „c OR $d^{d}$ ist nicht gleich "d OR c". Bei Aufgaben zur Booleschen Algebra und Logik ist das natürlich durchaus ein Problem! Hier liegt allerdings kein Bug im eigentlichen Sinne vor, denn die MAXIMA Bedienungsanleitung schreibt dazu ganz klar "AND ist nicht kommutativ, da aufgrund von nicht ausgewerteten Operanden die Ausdrücke $a$ AND b und b AND a ein unterschiedliches Ergebnis haben können."

Auch bei der Verwendung der imaginären Zahl i (in MAXIMA als \%i definiert) ist Vorsicht geboten. Der Ausdruck $1 / \mathrm{i}$ wird in MAXIMA nicht direkt als äquivalent zu -i interpretiert, sondern muss erst durch Nutzung der Funktion expand(1/\%i) "vereinfacht" werden, um äquivalent zu -i erkannt zu werden. Hier gäbe es weitere Beispiele basierend auf Besonderheiten von MAXIMA. Die Leistungsfähigkeit dieses Open Source Computeralgebrasystems ist enorm, aber für eine effektive und zuverlässige Verwendung muss man sich hier erst mit der "Technik" vertraut machen.

Bei der Klausurplanung sollten auch Nomenklaturen bspw. für Indizes genau diskutiert und auf Ihre Tauglichkeit geprüft werden. Das Formelzeichen $a_{i}$ würde in MAXIMA bspw. mittels a[i] eingegeben, hingegen im Textsatzsystem LaTeX als a_i. Bei numerischen Indizes, wie bspw. $a_{1}$ wäre in MAXIMA zudem die Schreibweise a1 zulässig. Bei der Vorschaufunktion in ONYX werden sowohl MAXIMA als auch LaTeX
Ausdrücke interpretiert, weshalb ein identisches Vorschaubild entsteht (Abb. 1) und die Eingabe damit für die Studierenden gleichwertig erscheint. Da MAXIMA allerdings nur die Schreibweise $a[i]$ als Index interpretieren würde, würde auch nur diese Antwort als korrekt bewertet. Bei Abfrage einer Variablen kann dieses Verhalten durch Hinterlegen von Alternativlösungen in ONYX abfangen werden, was jedoch bei Formelvergleichen nur aufwändiger zu lösen wäre. Um auch den Studierenden gerecht zu werden und nicht das Lehrziel MAXIMA mit in den Lehrplan aufzunehmen, können durch Schreibweisen wie ai bzw. Al auch Indizes unterbunden werden - in jedem Fall sollte hier aber ein durchgängiges und einheitliches Konzept in Aufgabenstellungen und Lösungen verfolgt werden.

$$
\mathrm{a} 1+\mathrm{a}_{-} 1+\mathrm{a}[1] \text { (?) } a_{1}+a_{1}+a_{1}{ }^{\otimes}
$$

Abb. 1: Vorsicht bei Indizes. Unterschiedliche Eingaben der Studierenden führen zu scheinbar gleichen Ergebnissen, die allerdings von ONYX und MAXIMA nicht gleich interpretiert werden.

Und wenn eine oder alle Aufgaben in ONYX plötzlich nicht mehr funktionieren, ist es ratsam zu prüfen, ob es eventuell gerade ein Server-Update gab, bei dem die ONYX oder MAXIMA Versionen aktualisiert wurden (so geschehen in der Prüfungsphase des Sommersemesters 2020, wodurch einige MAXIMA Berechnungen funktionsunfähig wurden). Hier wäre es wünschenswert, allen OPAL oder ONYX Nutzern im Voraus eine automatisierte Info über Updates zukommen zu lassen.

Aber auch richtige Bugs führen teilweise zu Funktionsproblemen, bspw. funktionierten Aufgaben von Typ Mehrfache Zuordnung bis Juli 2020 nur mit maximal vier Zeilen, obwohl man mehr Zeilen einfügen konnte. Oder aber bei Aufgaben vom Typ Grafische Zuordnung konnte man mehrere Elemente übereinander ziehen oder verlor Elemente durch einen Programmierfehler im digitalen Nirwana - hier reagierte BPS sehr schnell und konnte mit unseren Hinweisen den Bug im Juli beheben. Besonders nervenraubend ist es, wenn Aufgaben sich plötzlich nicht mehr speichern lassen bzw. 
die vermeintlich gespeicherten Änderungen nicht gespeichert werden (Abb. 2).

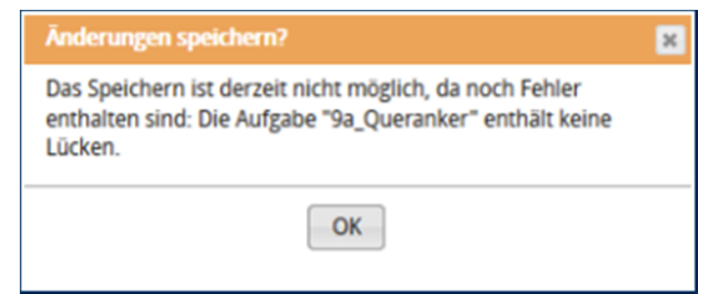

Abb. 2: Nervenraubende, weil nicht nachvollziehbare ONYX Fehler (es waren mehrere Lücken in der Aufgabe implementiert), die nur durch Neuerstellung der Aufgabe umgangen werden können.

Hier hilft aus unserer Erfahrung heraus im besten Falle ein Kopieren der Aufgabe oder leider häufiger nur ein vollständiges Neuerstellen. Und manchmal hilft bei ONYX nur ein Abmelden, Schlafengehen und Anmelden, damit Aufgaben über Nacht plötzlich funktionieren. Warum einige dieser Probleme auftreten und aufgrund welcher Umstände, konnten wir nicht immer klären. Laut BPS erfolgt wohl regelmäßig gegen 2:00 Uhr nachts eine automatische Bereinigung des Systems, was zumindest eine Erklärung für die teils wundersame Problemlösung über Nacht wäre.

Ein häufig wiederkehrender Wunsch bei unserem Autorenteam war ein Offline-Tool zur Testerstellung, aus dem Aufgaben oder eben ganze Tests dann exportiert werden können, um diese bspw. im ONYX Aufgabenpool zu importieren. Hintergrund dieses Wunsches ist, dass die verschachtelte Struktur, ein umständliches Erstellen von mehreren Variablen, lange Ladezeiten für Seiten und Instabilität (vermutlich durch hohe Serverlast) ein enormer Zeitfresser sind und die Nerven auf die Probe stellen.

Eine Klausur wird in OPAL als Kursbaustein Test verstanden. Die TU Dresden hat sich insbesondere aus Stabilitäts- und Datenschutzgründen entschieden, für Prüfungen eine eigene OPAL-Instanz namens OPAL Exam@TUD zu erstellen. Der Kursbaustein wird in der Regel mit einem ONYX Test verknüpft, welcher aus mehreren Aufgaben besteht. Zudem kann man als Strukturierungshilfe noch Sektionen einfügen. Wesentliche Auswirkungen ergeben sich bei einem Test durch die zu definierende
Bearbeitungsabfolge als linear oder nichtlinear. Bei einer linearen Abfolge der Aufgaben müssen die Studierenden die Prüfung also schrittweise im vorgegebenen Muster durchlaufen. Wünschenswert wäre daher eine nichtlineare Abfolge, die es den Studierenden ermöglicht, entsprechend ihrer Stärken und Schwächen eine individuelle Abfolge der Aufgaben zu wählen. Allerdings geht das schon dann nicht, wenn nur eine Aufgabe auf einer anderen aufbaut oder aber mit einer Aufgabe die Lösung zu einer vorhergehenden verraten würde. Der programmiertechnisch nicht so versierten Aufgabenersteller:in, scheint die einfache Lösung naheliegend, innerhalb einzelner Sektionen eine lineare Abfolge festzulegen, zwischen mehreren Sektionen allerdings nichtlinear, frei auswählen zu können - leider ist das derzeit nicht möglich. Um hier einen zufriedenstellenden Workaround zu schaffen, wurde die Klausur MAT2 daher innerhalb eines Kurselementes vom Typ Struktur in 12 Tests aufgeteilt, von denen jeder Test eine klassische Komplexaufgabe bzw. einen Themenblock darstellt. Zusätzlich wurde initial ein Test für die Selbständigkeitserklärung eingefügt. Um die Bearbeitungszeit nicht für jeden Test starr festzulegen und damit eine individuelle Zeiteinteilung zu erlauben, wurde der Zugang zum Kursbaustein Struktur auf die Bearbeitungszeit beschränkt. Soweit noch übersichtlich, allerdings kommt eine weitere Herausforderung zum Tragen - die Berücksichtigung unterschiedlicher Bearbeitungszeiten für Studierende mit Nachteilsausgleich. Leider war es bis zu Beginn 2021 nicht möglich, Studierenden ohne Abbruch der Bearbeitung (meist aufgrund von technischen Problemen) ein zusätzliches Zeitkontingent zuzuweisen. Hierfür wurde der Baustein Struktur daher vervielfältigt, um sowohl nach Nachteilsausgleich zu unterscheiden als auch nach einer HISQIS Registrierung (Abb. 3).

Der präsentierte Workaround hat aber auch seine Schattenseiten, denn bei der Ausführung der Klausur im Prüfungsmodus ist es bei technischen Problemen eines Studierenden erst notwendig zu wissen, in welchem Test gerade gearbeitet wurde, um bspw. ein Fortsetzen der Bearbeitung zu ermöglichen. Zudem muss immer wieder zwischen den Tests gesprungen werden, um mögliche Probleme mittels der 
Prüfungsansicht zu identifizieren. Darüber hinaus ergibt sich der erschwerende Nebeneffekt, dass beim Export der Klausurergebnisse nicht eine Datei sondern für unseren Fall $4 \times 12$ also 48 Einzeldateien exportiert und wieder miteinander harmonisiert werden müssen. Wünschenswert wäre folglich eine erweiterte Teststruktur, die eine Kombination linearer und nichtlinearer Inhalte ermöglicht.

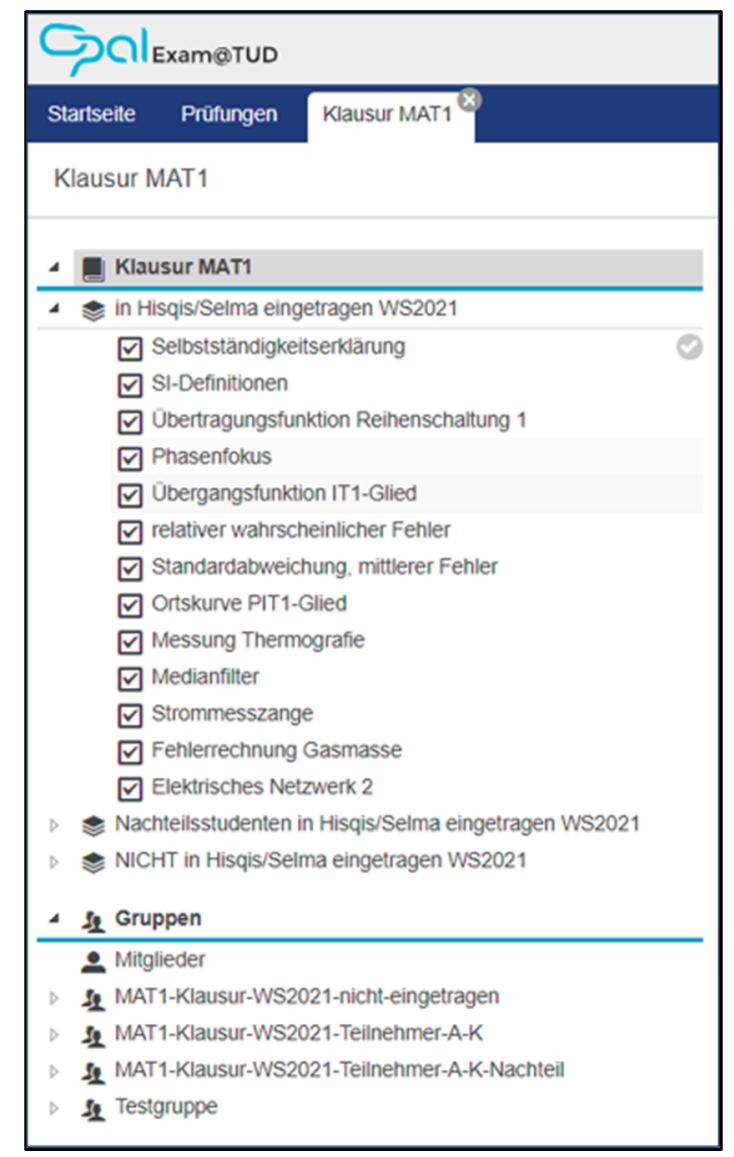

Abb. 3: OPAL "Struktur" Kursbausteine als Container für eine Klausur mit den Aufgaben als separaten linearen Tests zur Kombination linearer und nichtlinearer Abläufe.

Im Sommersemester 2020 überraschten bei der Arbeit in ONYX und OPAL noch viele Besonderheiten oder auch Bugs, die an BPS als Entwickler gemeldet wurden. Zudem wurden Wünsche zur Implementierung einiger fehlender Funktionalitäten geäußert. Wesentliche Punkte waren für uns hier einerseits die Möglichkeit zur individuellen Verlängerung der Bearbeitungszeit beispielsweise für Studierende mit Nachteilsausgleich (ohne, dass technische Probleme vorliegen) und andererseits die Möglichkeit zur Kombination linearer und nicht-linearer Teststrukturen. Insbesondere dieser letzte Punkt ist wesentlich für eine Akzeptanz und Gleichwertigkeit von Online-Prüfungen. Studierende müssen entsprechend ihrer persönlichen Stärken die Möglichkeit haben, sich die Bearbeitungszeit sowie die Bearbeitungsinhalte über eine nichtlineare Struktur eines Tests individuell einteilen zu können. Dementgegen steht, dass insbesondere anspruchsvollere, komplexe Aufgaben als reine Online-Varianten nur mit aufeinander aufbauenden, linear strukturierten Teilaufgaben umsetzbar sind (ohne Lösungen von Teilaufgaben auf dem Silbertablett zu liefern).

Erfreulich ist, dass die individuelle Zeitvorgabe von BPS noch kurz vor der Prüfungsperiode im Frühjahr 2021 implementiert wurde. Allerdings nur im Rahmen eines Tests. Für OPALKursbausteine vom Typ Struktur, in denen mehrere Tests zusammengefasst sind, siehe Abb. 3, ist das nicht möglich. Erneut und deshalb überaus ärgerlich ist, dass die fehlende Kombination linearer und nichtlinearer Teststrukturen die Arbeit der Aufgabenersteller:innen so massiv beeinträchtig! Für uns ist das die zentrale Schwachstelle der aktuellen ONYX Test-Suite.

Hieraus resultierte leider auch, dass die implementierte individuelle Zeitvorgabe faktisch nicht nutzbar war und der bekannte, deutliche Mehraufwand wieder entstand, da ein rein linearer Prüfungsablauf nach unserer Wahrnehmung keine faire Prüfungslösung darstellt. Um eine nichtlineare Klausur bereitstellen zu können, die gleichzeitig lineare Komplexaufgaben enthält, wurden daher erneut alle Aufgaben als eigenständige Tests innerhalb eines übergeordneten OPAL Kursbausteins Struktur definiert. Damit hatte eine individuelle Zeiterhöhung innerhalb eines ONYX Tests leider keine Auswirkung auf einen anderen Test im gleichen Strukturbaustein von OPAL, womit die neu implementierte Funktion für unseren Fall leider ihre eigentliche Aufgabe völlig verfehlte. Anstelle eines ONYX Tests mit 13 Aufgaben hatten wir also wie im Vorsemester einen OPAL Kursbaustein Struktur als Klausur mit 13 linearen ONYX Tests als Aufgaben (vgl. Abb. 3). Da wir zudem für Studierende mit Nachteilsausgleich eine erhöhte Bearbeitungszeit realisieren mussten, musste diese Klausurstruktur in OPAL wieder gedoppelt werden und zusätzlich benötigten wir eine weitere Struktur 
für Studierende ohne HISQIS bzw. SELMA Eintrag, sodass sich schließlich insgesamt 39 ONYX-Tests ergaben.

Um den Studierenden eine ergänzende Sicherheit zu bieten und bei unklaren Fehlern eine weitere Entscheidungsgrundlage zu haben, wurde nach rechenintensiven Teilaufgaben eine Uploadaufgabe integriert, in der die Studierenden handschriftliche Notizen oder Skizzen hochladen können (Abb. 4). Leider führten die Dateiuploads unter Prüfungsbedingungen teilweise zu deutlichen Verzögerungen.

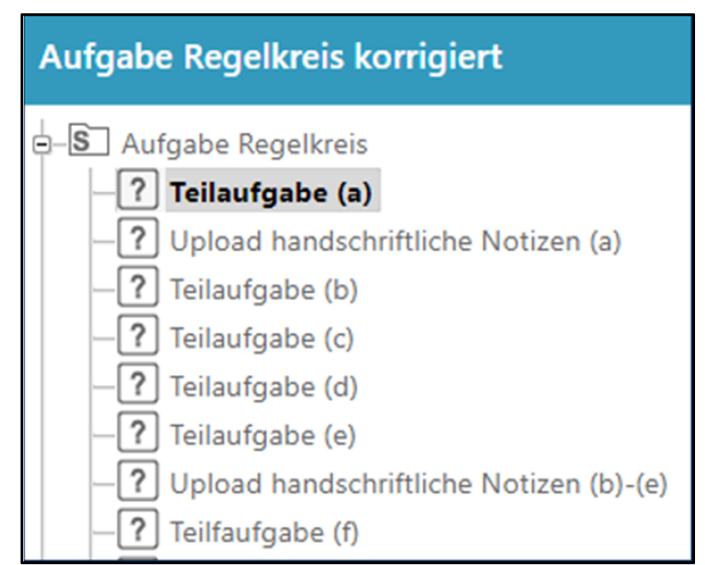

Abb. 4: In einen Test integrierte Aufgaben zum Upload handschriftlicher Notizen nach rechenintensiven Teilaufgaben.

\section{Befähigung der Studierenden}

Wie bereits erläutert, muss die Syntax von MAXIMA erlernt werden - natürlich auch von den Studierenden. Daher ist es essentiell, frühzeitig im Semester Tests anzubieten, mit denen eine Eingabe von Formeln in MAXIMA-konformen Ausdrücken, aber auch der Umgang mit OPAL, ONYX, MAXIMA und Feedbacksystemen sowie der eigenen Hardware geübt werden kann.

Wir haben zusätzlich versucht, diese SyntaxProblematik dadurch zu entzerren, dass wir eine einfache Schreibweise für Variablen mit Indizes (bspw. $\mathrm{DT}=\mathrm{D}_{\mathrm{T}}$ ) vorgegeben haben, die in den betreffenden Aufgaben als Begleittext über eine globale Variable im Test mit in die Aufgabenstellung eingebunden wurde. Trotzdem traten hier während der Klausur mehrfach Nachfragen auf, weshalb auf semsterbegleitende Tests nicht verzichtet werden sollte.
Auch der Upload von Dateien, das Erfassen (scannen, fotografieren) und komprimieren von Dateien oder eine realistische Einschätzung der Stabilität der heimischen Internetverbindung sind relevant.

Wünschenswert wären dementsprechend fakultätsübergreifende, einheitliche Regeln für OPAL, ONYX und MAXIMA. Eventuell könnten den Studierenden perspektivisch Einführungskurse für diese Werkzeuge mit Tipps und Anregungen für die Heimarbeit und Online-Prüfungssituationen angeboten werden. So könnten Studierende und Lehrende auf einem Grundstock aufsetzen, der für alle Veranstaltungen und Lehrstühle im Wesentlichen identisch wäre.

\section{Prüfungsbetreuung}

Die Begleitung und Betreuung der Studierenden während einer Prüfung ist bei digitalen Formaten von enormer Bedeutung und scheint aus unserer Sicht zwingend erforderlich für eine faire und erfolgreiche Online-Prüfung. Fragen oder Probleme der Studierenden können so geklärt oder auch mögliche Fehler in Aufgaben schnell identifiziert werden. Die Rückmeldungen der Studierenden bestätigen, dass allein die Möglichkeit Probleme oder Fragen an die Betreuenden richten zu können, Sicherheit gäbe und beruhigend wirken würde man sei nicht allein während der Prüfung. Das ist ein nicht zu unterschätzender Wert und positiver psychologischer Effekt.

Die Vorbereitungsphase bis zur fertigen Online-Klausur ist lang und intensiv - alle Daten werden zigmal überarbeitet, auf Verständlichkeit und inhaltliche Korrektheit geprüft. Dann kommt die Klausur und (natürlich) finden Studierende einen Fehler in der Aufgabenstellung! Was tun? Alle Studierende müssen nun schnellstmöglich über das Vorgehen informiert werden, aber wie?

Begleitend zu den OPALexam Servern moderieren wir einzelne Chaträume mit jeweils ca. 80 Personen bei der Element-Instanz der TU Dresden (matrix.tu-dresden.de), welche auf dem quelloffenen Messenger-Protokoll Matrix basiert. Die Plattform hat sich dabei über zwei Semester und mehrere Prüfungen sowie Praktika als sehr zuverlässig, schnell und effizient 
für die interne Kommunikation sowie die Prüfungsbetreuung erwiesen.

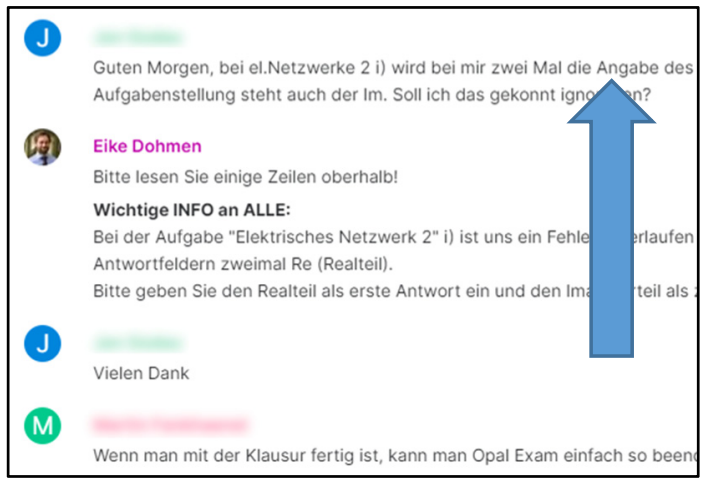

Abb. 5: Auszug aus einem prüfungsbegleitenden Chatraum: Was tun, wenn bei einer Online-Klausur Fehler entdeckt werden?

Die Chats sind prädestiniert, um wesentliche Informationen nahezu instantan an alle Studierenden zu verteilen. Allerdings "wandern" auch wesentliche Informationen aufgrund der Chatstruktur schnell aus dem Blick und müssen daher regelmäßig erneut gepostet werden, um niemanden zu benachteiligen (Abb. 5).

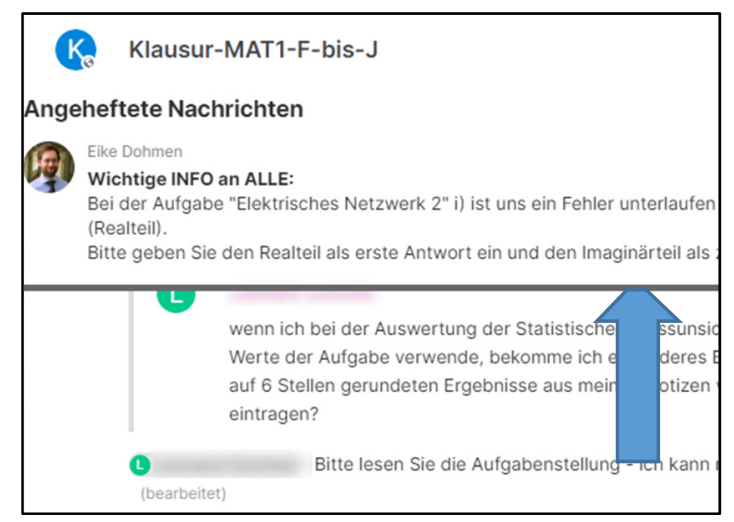

Abb. 6: Blick in die Zukunft: mit der Labor-Funktion „Anheften" können wichtige Nachrichten für alle Mitglieder eines Raums dauerhaft im Blick bleiben.

Während der MAT 1 Klausur in diesem Wintersemester konnten so, unter Nutzung des Matrix-Chats, 99\% alle Studierenden erfolgreich auf einen Fehler in der Aufgabenstellung hingewiesen werden. Um die dauerhafte Bereitstellung wesentlicher Informationen im Chat zu ermöglichen, gibt es derzeit in Matrix im Labor-Stadium die Funktionalität Anheften, wodurch Nachrichten direkt unterhalb vom Raumnamen fest angeheftet werden (Abb. 6).
Dieses Feature soll perspektivisch auch für alle anderen Benutzer zur Verfügung stehen.

\section{Absicherung durch Datei-Upload}

Neben der aktiven Betreuung ist die Möglichkeit zum Upload bspw. von handschriftlichen Notizen oder Skizzen ein hilfreiches Instrument, um Studierenden eine ergänzende Sicherheit zu bieten und bei unklaren Fehlern während der Korrektur eine Entscheidungsgrundlage zu schaffen. Dementsprechend wurde nach rechenintensiven Teilaufgaben eine Uploadaufgabe in die Tests integriert. Leider sind die Dateiuploads ein wesentlicher Grund für eine erhöhte Serverlast und benötigen teilweise relativ viel Zeit, welche den Studierenden unter Prüfungsbedingungen dann gegebenenfalls fehlt. Abhilfe würde hier ein Upload nach der Klausur schaffen, der dann allerdings die Wahrscheinlichkeit von inkonsistenten Antworten und von Täuschungsversuchen erhöht.

\section{Multi-Tasking für die Prüfenden}

Zu Beginn der Prüfungsphase des Sommersemesters 2020 zeigten sich teils deutliche Leistungsengpässe von OPAL, welche insbesondere bei großen Klausuren mit viel "Gleichzeitigkeit" von Nutzer:innenaktionen (wie beispielsweise Uploads von Ergebnissen) zu langen Wartezeiten und in einem Fall sogar zum Serverabsturz während einer laufenden Prüfung führten. Dementsprechend wurde noch während der Prüfungsperiode im Sommer 2020 eine schnelle und einfache Lösung zur Behebung umgesetzt, indem drei unabhängige Instanzen von OPAL ausschließlich für Prüfungen mit dem Namen OPALexam auf Servern an der TU Dresden eingerichtet wurden. Leider blieb diese Notlösung auch noch für die Prüfungszeit im Frühjahr 2021 erhalten. Hierdurch mussten die Betreuenden neben ggf. vorhandenen Kommunikationsplattformen auch auf drei separaten Servern gleichzeitig eingeloggt sein, um die Studierenden während der Klausur betreuen zu können (Abb. 7). Zudem konnten Verbindungsprobleme, fehlende Einschreibungen oder sonstige technische Probleme bei Studierenden nur unter exakter Kenntnis des Vor- und Nachnamens (sonst uneindeutig), Kenntnis über einen möglichen Nachteilsausgleich sowie Kenntnis der 
zuletzt bearbeiteten Aufgabe behoben werden.

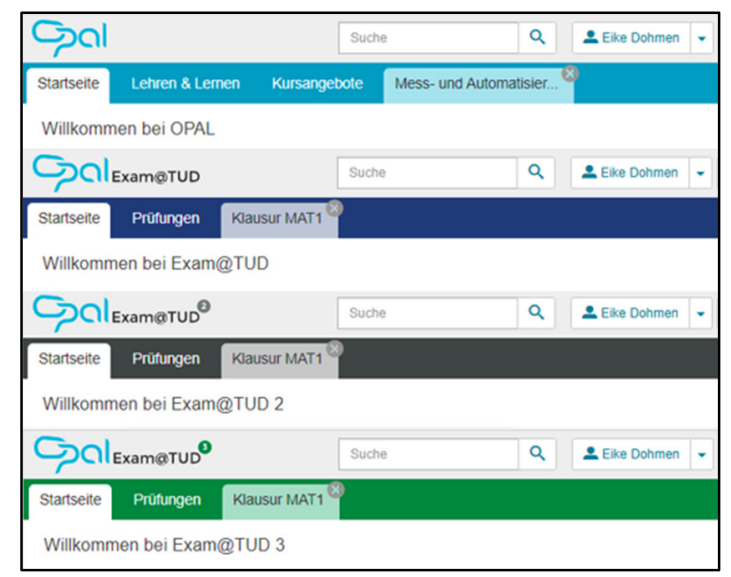

Abb. 7: OPAL, OPALexam, OPALexam2 und OPALexam3 - hier ist Multitasking der Prüfenden gefragt.

Durch diese parallelen Strukturen mussten die Betreuer gleichzeitig auf drei Servern mit jeweils drei der genannten OPAL-Kursbausteine vom Typ Struktur (mit/ohne Nachteilsausgleich, mit/ohne HISQIS/SELMA Eintragung) mit wiederum jeweils 13 ONYX Tests aktiv sein und nebenher noch zwei Kommunikationskanäle (Betreuende untereinander, Betreuende mit Studierenden) bedienen. Wollte Studierenden „mal schnell“ das Fortsetzen der Bearbeitung ermöglicht werden, musste - wie bereits beschrieben - zuerst der vollständige Name, der Server, der jeweilige Struktur Kursbaustein und die zuletzt bearbeitete Aufgabe bekannt sein.

\section{Prüfungssteuerung}

Unter dem Begriff Prüfungssteuerung ist ein sehr hilfreiches Werkzeug zur Kontrolle der Prüfung und zur Lösung von Problemen in OPAL integriert worden. Hier hat BPS in 2020 und 2021 viele Verbesserungen implementiert, wie beispielsweise die Anzeige der individuell verbleibenden Restzeit von Studierenden oder aber die Anzeige von Verbindungsproblemen sowie eine automatische Fortsetzung der Prüfung nach Verbindungsabbrüchen. Trotzdem sind auch hier noch Verbesserungen möglich. Einige ausgewählte Besonderheiten bzw. Kuriositäten stellen wir im Folgenden vor.
Teilweise schien es leider nicht möglich nach Ablauf der Bearbeitungszeit die Klausur manuell für wenige noch bearbeitende Studierende zu beenden (Abb. 8). Wir vermuten, dass es sich hierbei um einen Anzeigefehler handelt und hoffen auf Aufklärung/Abhilfe durch BPS.

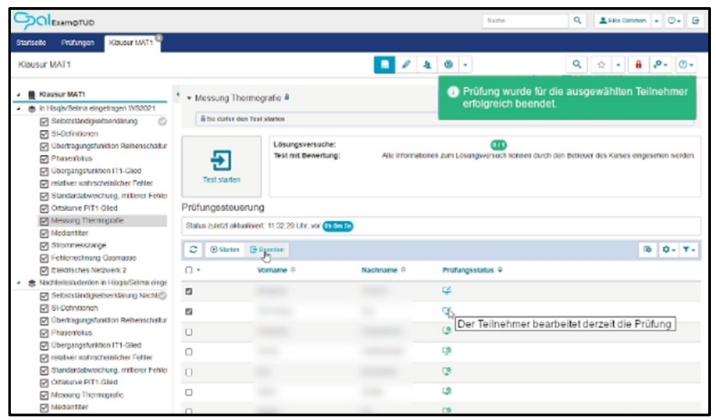

Abb. 8: Screenshot vom Ende der Klausur - nach Ablauf der Bearbeitungszeit (inkl. evtl. gewährter Zeitpuffer) wurden einige Studierende weiterhin als „bearbeitend" angezeigt; kurios - auch mit der Prüfungssteuerung konnte dieser Status nicht geändert werden.

Einige wenige Studierende hatten bei unserer Probeklausur mit einer Bearbeitungszeit von $1 \mathrm{~h}$ nach einer $1 \mathrm{~h}$ noch über $1 \mathrm{~h}$ Restzeit für die Bearbeitung, was nach unserem Verständnis unmöglich sein müsste (Abb. 9). Bei diesen Einzelfällen musste (und konnte) die Probeklausur manuell beendet werden.

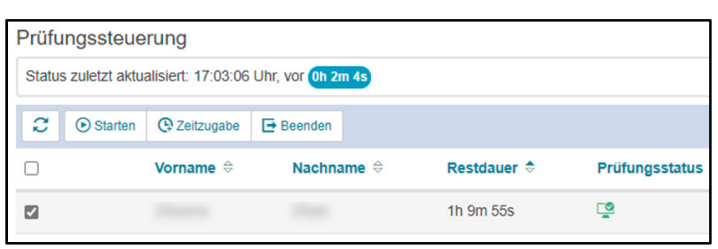

Abb. 9: Screenshot vom Ende der Probeklausur ein Student hat noch $1 \mathrm{~h} 10 \mathrm{~m}$ Restdauer für die Bearbeitung, obwohl die Probeklausur nur $1 \mathrm{~h}$ Bearbeitungszeit hatte.

\section{Prüfungszeitraum variabel?}

Ein Student, der die Prüfung erst kurz vor dem Bearbeitungsende um 10:30 Uhr startete, reagierte sehr verärgert auf das harte, manuelle Ende der Klausur um 11:15 Uhr und postete einen Screenshot, der den Grund für seine Entrüstung offenbarte: bei SELMA (dem OnlinePortal zur Prüfungsverwaltung) wurde der Klausurzeitraum fälschlicher Weise von 00:05 Uhr bis 23:59 Uhr aufgeführt (Abb. 10). 


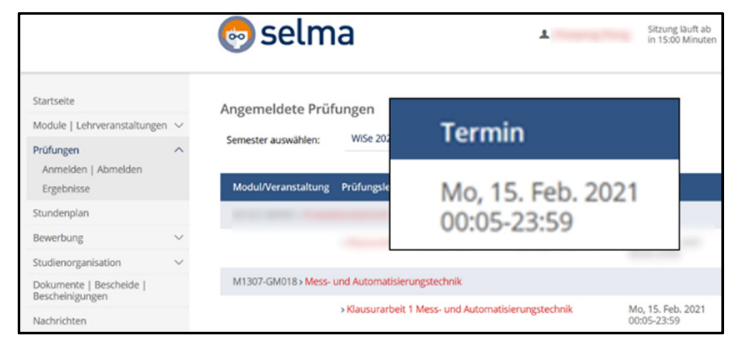

Abb. 10: Screenshot eines Studenten aus SELMA als Prüfungszeitfenster ist der ganze Tag ausgewiesen.

\section{Datenexport}

Das Prüfungsteam war außerordentlich erleichtert, als die Klausur mit über 500 Studierenden ohne größere Zwischenfälle erfolgreich beendet wurde. Traditionell wird dann an unserem Lehrstuhl noch am gleichen Abend eine erste Übersichtsstatistik zur Punkteverteilung des in Präsenzklausuren üblichen Fragenteils veröffentlicht.

Die sehr ungünstige Zerstückelung der Klausur über mehrere Server und Substrukturen zieht sich leider besonders merklich bis in die Phase des Datenexports und der Prüfungskorrektur. Nun mussten alle Ergebnisse aus 13 Tests in 3 Kursbausteinen Struktur auf 3 unabhängigen Servern - folglich 117 Einzeltests - heruntergeladen, geprüft und ggf. nachbewertet werden.

Allein für die entsprechende Datenarchivierung aus OPAL inkl. Download der 3,8 GB ZIPArchive wurde (bei guter Internetverbindung) fast eine Stunde benötigt! Danach mussten die Excel-Dateien mit den Testergebnissen aus den Archiven extrahiert werden, was leider immer noch schneller war als ein alleiniger Download der Excel-Files in OPAL (denn es gibt lediglich eine Möglichkeit zur Anzeige der PDFDatei, d. h. jede PDF-Datei muss erst angezeigt werden, um diese anschließend speichern zu können. Nun hatte einer der Autoren die ehrenvolle Aufgabe alle 117 Excel-Dateien zusammen zu kopieren, um zumindest danach eine effiziente Korrektur zu erzielen. Grundsätzlich ging das mit 2-3 Arbeitsstunden verhältnismäßig schnell, ...

\section{Voreinstellungen und Zeichensätze}

...wäre da nicht noch die Tatsache, dass auf allen genannten drei OPALexam Instanzen (OPALexam, OPALexam2 und OPALexam3)
VOR dem Export noch die Exporteinstellungen hätten einheitlich auf seine persönlichen Einstellungen gesetzt werden müssen, um auch immer Excel-Dateien mit passender Zeichenkodierung zu erhalten (Abb. 11).

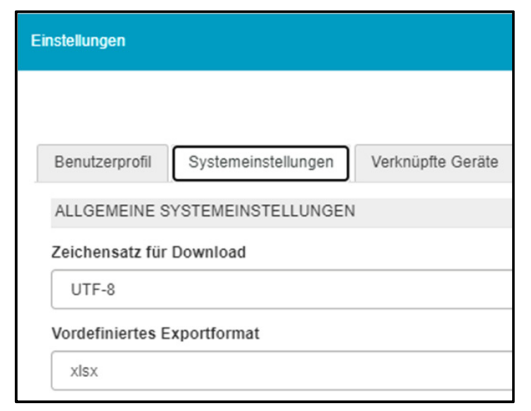

Abb. 11: Systemeinstellungen zum Exportformat sowie zum Zeichensatz.

Konkret waren auf den drei OPALexam Instanzen drei verschiedene Grundeinstellungen für das Exportformat festgelegt - anscheinend werden diese Grundeinstellungen also weder vom vorhandenen OPAL-Account übernommen noch einheitlich gesetzt. Probleme entstehen daraus ggf. bei der Datenarchivierung (manchmal CSV, manchmal XLS) und vor allem bei der Erkennung von Zahlen, die je nach Zeichensatz als Text interpretiert werden!

Alle Zahlen wurden bei uns als Text eingelesen, was wiederum bedeutete, dass alle Spalten mit Zahlen nachträglich, EINZELN in Zahlen konvertiert werden mussten (Text in Spalten). Damit aber nicht genug, denn beim Export der Ergebnisse der MAXIMA-Rechenaufgaben wurden die Lernerantworten entgegen der sonstigen Einstellung mit "." als Dezimaltrennzeichen exportiert. Hier sind beim Datenexport einige Fallstricke verborgen, die sich in Luft auflösen würden, wenn nur eine OPAL-Instanz mit einem ONYX Test (für eine Klausur) und einer global gültigen, einfachen Voreinstellung, deren Auswirkungen auch dem Laien verständlich sind, ausreichend wären. Der Aufwand zur Softwareanpassung durch BPS sollte daher immer auch der Tatsache gegenübergestellt werden, dass viele Nutzer:innen mit den dargestellten Mehraufwendungen zu ringen haben.

\section{Datenverluste?}

Mit der zusammengeführten Tabelle ist dann ein wesentlicher Schritt zur Kontrolle und Nachbewertung gemacht. Teilweise lagen in 
der Excel-Tabelle aus der Datenarchivierung aber keine Variablenwerte vor oder diese wurden als „null“ deklariert (Abb. 12).

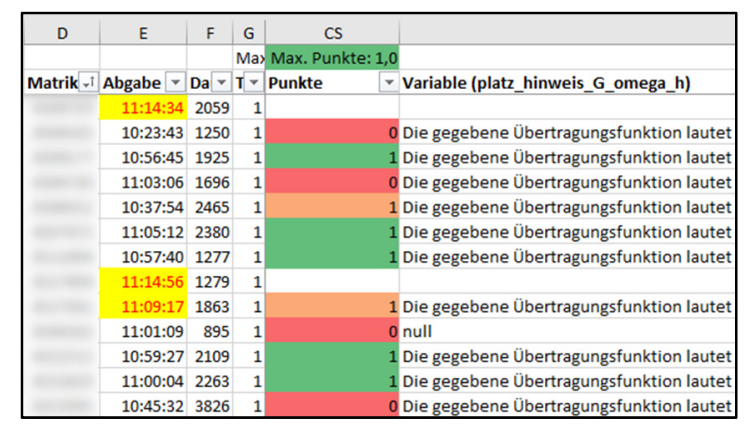

Abb. 12: Auszug aus der erstellten Excel-Bewertungstabelle mit fehlenden Variablenwerten (rechts).

Positiv ist, dass einerseits die Punktzahlen korrekt in die Excel-Datei exportiert werden und dass andererseits die Prüfungsansicht im Webinterface neben den Eingaben der Studierenden auch deren Sicht der Prüfung vollständig dokumentiert und als PDF bereitstellt.

Ein ähnliches Problem tritt beim Export von Gleitkomma-Variablen (Float) aus Aufgaben mit MAXIMA-Berechnungen auf, bei dem nur 2 Nachkommastellen exportiert werden. In einer Fehlerrechnungsaufgabe wurde damit die Variable delta_p_global_e nicht wie angegeben mit 1,140762589549 exportiert, sondern landete in Excel als 1,14. Da auf Basis dieser Variablen weitere Berechnungen angestellt werden, welche mit 6 Nachkommastellen korrekt angegeben werden müssen, hilft dann nur noch eine Analyse im Webinterface. Problematisch ist das vor allem, wenn eine Auswertungsformel fehlerhaft angegeben wurde und für alle Studierenden eine Nachberechnung der Ergebnisse durchgeführt werden soll - hier fehlen dann die entsprechenden Eingangsdaten.

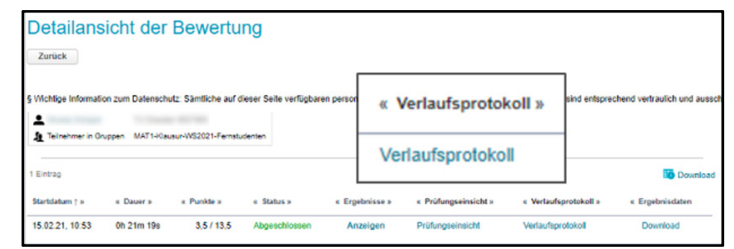

Abb. 13: Verlaufsprotokoll als neues, mächtiges Feature in OPAL zur Prüfungsbewertung
Da es zudem bisher nicht möglich ist, solche Fehler der Prüfenden durch eine automatisierte Neuauswertung im Webinterface abzufangen, müsste hier jede Variable manuell exportiert werden, um nachbewerten zu können. Mit dem Verlaufsprotokoll hat BPS ein mächtiges Werkzeug implementiert, um auf Basis der Server-Protokolle Problemen auf die Schliche zu kommen (Abb. 13).

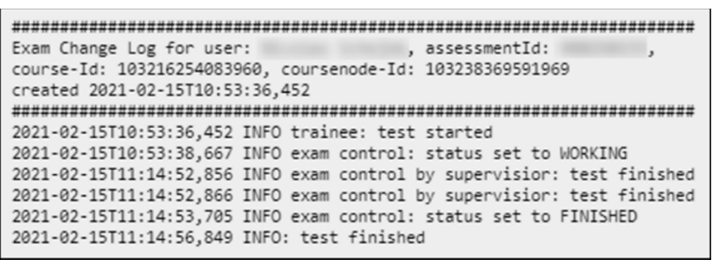

Abb. 14: Problemanalyse mit dem Verlaufsprotokoll - Prüfungsabbruch durch Prüfer (Ende Klausur 11:00 Uhr - Beendigung durch Prüfer im übergeordneten OPAL-Kursbaustein Struktur für alle verbleibenden Teilnehmer 11:14:52 Uhr).

Bezüglich des oben genannten Problems mit fehlenden Variablen in der Exportdatei konnte so festgestellt werden, dass die leeren Zellen immer bei einem Prüfungsabbruch durch Prüfende oder Studierende auftraten (Abb. 14).

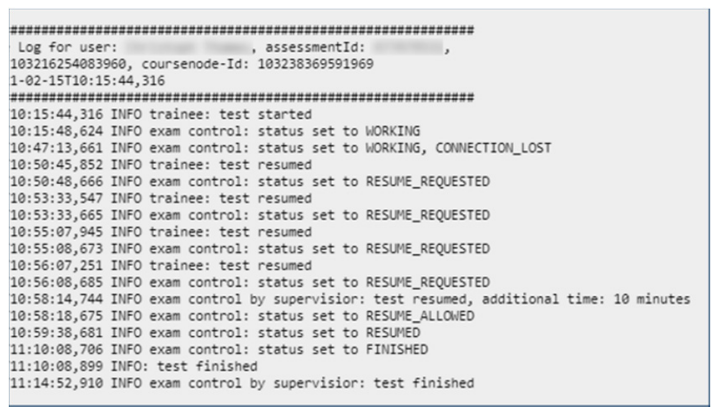

Abb. 15: Problemanalyse mit dem Verlaufsprotokoll - Zeit abgelaufen (Ende der Klausur mit 10 Minuten Zusatzzeit 11:10 Uhr).

Für Zeilen, die lediglich „none“ als Variablenwert konnte hingegen ein Ablauf der Prüfungszeit während der Bearbeitung als Ursache identifiziert werden (Abb. 15).

\section{Prüfungskorrektur}

Im Rahmen der Neuerstellung unserer Klausur hatten wir uns stets bemüht, die Perspektive der Studierenden zu berücksichtigen. Bei der Korrektur der Klausur wurde dann klar, dass 
auch der Blick auf die Aufgaben aus der Perspektive der Korrigierenden notwendig ist. Welche Aufgaben sind effizient zu korrigieren und zu bewerten? Wo geraten wir in Entscheidungsprobleme? Ein Beispiel hierfür ist die klassische Lückentextaufgabe, bei der neben dem Fachwissen der Studierenden auch deren Wortschatzwissen, Satzbildung, Mundart, vorhandene Tastatur und nicht zuletzt die Rechtschreibung zu berücksichtigen sind. So wurden bspw. für eine Lücke mit der korrekten Antwort "additiv" folgende Antworten gegeben, die als korrekt zu bewerten oder zumindest zu diskutieren wären.

$\begin{array}{ll}\text { - additiv } Y+Z=Y 2 & \text { - verknüpft } \\ \text { - addiert } & \text { - gekoppelt } \\ \text { - addierend } & \text { - zusammen } \\ \text { - addierent } & \text { - zusammengeführt } \\ \text { - per Addition } & \text { - kombinierend } \\ \text { - summarisch } & \text { - konjunktiv } \\ \text { - vereinigt } & \text { - positiv }\end{array}$

Diese Menge an korrekten Antworten für einen einfachen Lückentext ist kaum vorhersehbar oder abfangbar. Mit einer Umstellung von einer Lückentextaufgabe auf eine Textboxaufgabe könnten diese Probleme abgestellt werden, ohne den Anspruch oder den Wert der Aufgabe wesentlich zu beeinflussen. Bei einer anderen Aufgabe informierte uns ein Student, er habe eine Schweizer Tastatur und daher kein " $\beta^{\prime \prime}$, weshalb er bei den betreffenden Antworten "ss" verwendet habe. Dieser "Fehler" wäre relativ gut durch Alternativlösungen abfangbar, aber auch hier ist die Nutzung einer Textboxaufgabe eine zuverlässige Alternative.

Ähnlich unvorhersehbar ist die kreative Intelligenz der Studierenden, die eine weitere korrekte Lösung bei einer vermeintlich eindeutigen Zuordnungsaufgabe generiert (Abb. 16). Bei der Bewertung von Aufgaben muss daher ergänzend zur automatisierten Auswertung immer noch eine manuelle Überprüfung bzw. Korrektur der Antworten erfolgen.

Bei Abbruch eines Tests während der Bearbeitung der letzten Aufgabe (bspw. durch technische Probleme) kann es vorkommen, dass die automatisierte Bewertung scheitert, da zufällig generierte Variablen für die Aufgabensteuerung nicht gespeichert werden. In unserer
Klausur passierte dies bei mehreren Studierenden bei einer Komplexaufgabe (realisiert über einen Test mit mehreren Aufgaben) zur Steuerung, bei welcher der Variablen \{Aufgabentyp\} zu Beginn des Tests zufällig "Taktstrasse" oder "Mischer" zugewiesen wurde.

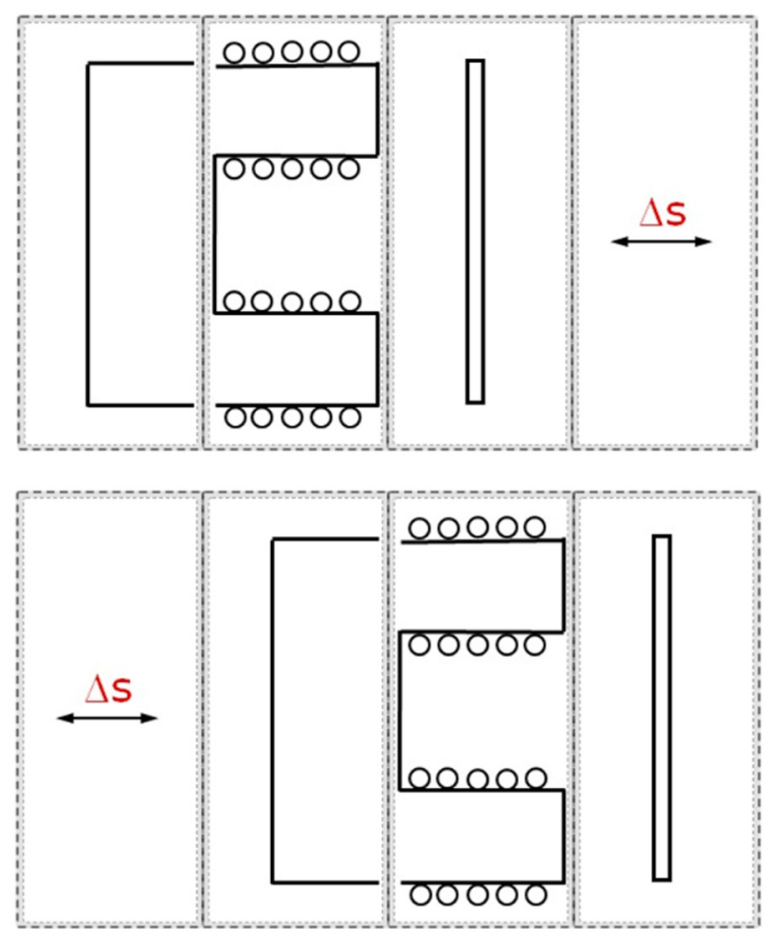

Abb. 16: Musterlösung (oben) und korrekte Alternativlösung (unten) zur Aufgabe: "Skizzieren Sie das Messprinzip eines induktiven Queranker-Wegaufnehmers".

Im beschriebenen Fall war die Variable \{Aufgabentyp\} allerdings leer, obwohl einige Studierende bereits alle Fragen auf Basis dieser Variablen beantwortet hatten. Die Studierenden erhielten damit null Punkte auf Ihre Antworten, unabhängig davon, ob diese richtig oder falsch waren. Dieser Fehler ist nur durch eine Nachkontrolle der Antworten identifizierbar. Hilfreich erwies sich hierbei der Datenexport in eine Excel-Datei, in welcher mit bedingter Formatierung falsche Lösungen farblich hervorgehoben werden konnten und auch komplexe Nachbewertungen auf Basis von Formeln möglich waren. Formelbasierte Nachbewertungen waren insbesondere bei systematischen Fehlern hilfreich, bspw. WENN (Aufgabentyp = "Taktstrasse") UND (Punkte $=0$ ) DANN (Zusatzpunkte $=+0,5$ ). Bezüglich des Datenexports ist noch anzumerken, dass das Exportformat (ins- 
besondere der Zeichensatz) in den persönlichen Einstellungen von OPAL festgelegt werden können (Abb. 11).

Ein Wermutstropfen beim Excel-Export ist derzeit noch, dass online getätigte Nachbewertungen inkl. der hinterlegten Begründungen nicht exportiert werden, weshalb derzeit eine kombinierte Online- und Offline-Bewertung nur bedingt möglich sind. Dieser Sachverhalt ist allerdings bereits an BPS übermittelt und wird hoffentlich bei den nächsten Überarbeitungen berücksichtigt.

Vorteilhaft ist die Online-Korrektur bei einer intensiven Prüfung jeder einzelnen Antwort aller Studierenden, da hier den Korrigierenden ein eindeutiges, übersichtliches optisches Feedback mit allen relevanten Informationen gegeben wird (Abb. 17). Nachteilig sind allerdings die Reaktionsgeschwindigkeit von OPAL zum Laden der Seiten und die verschachtelte Navigation. Leider wird das optische Feedback beim PDF-Export (bspw. mit der Funktion Archivieren) nicht mit exportiert. Die zentral in ein Archiv exportierten PDFs lassen sich zwar viel schneller öffnen und sichten, allerdings fehlt hier leider dieses sehr hilfreiche Feature. Positiv zu bemerken ist, dass BPS hier stetig die Schnittstellen weiterentwickelt und auch an dieser Stelle bereits einige neue Features seit der Klausur ergänzt wurden.

Fehler passieren und können nur durch intensive, kritische Überprüfungen identifiziert werden. Gerade durch den komplexen und für die Bediener:innen nur bedingt transparenten Aufbau von OPAL und ONYX sind Flüchtigkeitsfehler kaum zu vermeiden. Inhaltliche Fehler können nur von Fachkolleg:innen effizient ermittelt werden. Um aber technische Fehler oder Probleme bei Klausuraufgaben zu identifizieren, wäre ein stochastischer Prüfungstestbot (ein Programm, das für eine definierte Anzahl von fiktiven Nutzer:innen zufällig Ergebnisse generiert) eine aus unserer Sicht denkbare Anregung. Dies könnte ein Programm sein, welches bspw. für 100 virtuelle Prüflinge Fragen mit Zufallswerten beantwortet, Tests abbricht oder Fragen überspringt usw. und die entstandenen Daten als Validierungsdatensatz dann exportiert.

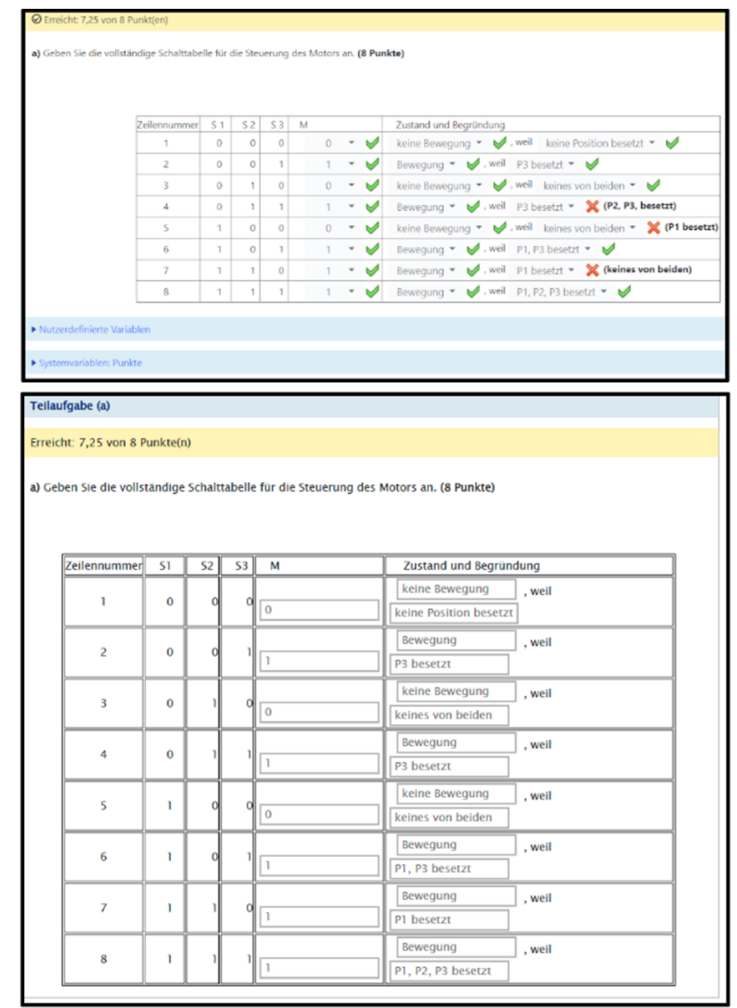

Abb. 17: Online-Bewertungswerkzeug mit optischem Feedback zu den Antworten inkl. der Musterantwort und Variablen (oben) im Vergleich mit dem entsprechenden exportierten Offline-PDF (unten).

\section{Täuschungsversuche}

Interessant ist die nähere Betrachtung der Dateinamen der Dateiuploads. Einerseits finden sich hier Hinweise auf zweifelhafte Aufnahmequellen (bspw. WhatsApp_Image_xyz) und andererseits erstaunliche Übereinstimmungen (bspw. „IMG20200728_081613_4.jpg“). Betrugsversuche konnten nicht ausgeschlossen werden, jedoch war unsere Wahrnehmung, dass sehr wenige Studierende versucht haben zu täuschen und insbesondere im Sommersemester 2020 der überwiegende Teil der Studierenden eigentlich eher froh war, überhaupt eine Prüfung ablegen zu können.

Im folgenden Wintersemester wurde jedoch deutlich häufiger behauptet, man habe sich gerade vertippt oder verklickt, hätte aber in den danach hochgeladenen handschriftlichen Notizen Belege dafür, dass man etwas ganz anderes abgeben wollte. Hier ist eine durchgängig eindeutige Vorgehensweise essentiell, die bereits vor der Klausur in Form von Rahmenbedingungen eindeutig kommuniziert wird - in diesem konkreten Fall, dass nur die Online-Ein- 
gabe zählt und die Uploads lediglich als Ergänzung bei technischen Problemen oder in Ausnahmefällen dienen.

\section{Potentialabschätzung}

Um Chancen und Grenzen darstellen zu können, möchten wir den Arbeitsaufwand abschätzen. Eine erste (fundierte) Prüfungsstatistik konnte bereits wenige Stunden nach der Prüfung generiert werden. Die Korrektur des gesamten Fragenteils dauerte in Summe etwa drei Personentage im Vergleich zu etwa einem Personenmonat bei der klassischen Papierklausur. Die Korrektur der Rechenaufgaben dauerte je Aufgabe lediglich zwei bis drei Personentage im Vergleich zu je einem halben Personenmonat. In Summe lag der Bewertungsaufwand damit bei 12 Personentagen im Vergleich zu bisher drei Personenmonaten (ca. 60 Personentage) je Semester. Dabei sind der Datenexport und die Datenkonsolidierung bereits anteilig mit einbezogen. Unabhängig vom enormen Einarbeitungs- und Vorbereitungsaufwand konnte die reine Korrekturzeit für die Klausur MAT2 damit auf 20\% reduziert werden. Der Vorbereitungsaufwand hingegen umfasste schätzungsweise 6 Personenmonate bei der initialen Umsetzung und etwa 1-2 Personenmonate im Folgesemester. Damit ist bei der Vorbereitung insgesamt ein deutlicher zeitlicher Mehraufwand von bis zu 100\% entstanden. Die Aufwandsabschätzung verdeutlicht das besondere Potential von Online-Prüfungen insbesondere durch Verlagerung der Zeitaufwendungen von der Korrektur in die Vorbereitung lernzielangepasster, aktueller Fragestellungen sowie einer Flexibilisierung des Lernens bei einer erhöhten Barrierefreiheit. Für die Lehrenden sinkt insbesondere bei Klausuren mit vielen Teilnehmenden (> 30 Personen) der Korrekturaufwand dramatisch. Aber auch für kleinere Lehrveranstaltungen kann sich der Aufwand lohnen, da gerade semesterbegleitende Lernstandüberprüfungen auf ähnliche Weise mit OPAL, ONYX und MAXIMA umgesetzt werden können und dann in leicht abgewandelter Form als Basis für eine Klausur dienen können. Zudem fällt der Einarbeitungsaufwand in Technik und Möglichkeiten nach der ersten Prüfungsgenerierung deutlich geringer aus, wodurch sich der Zeitaufwand für die Klausurerstellung sowie durchführung erneut reduziert.

\section{OPAL \& ONYX}

Die von der TU Dresden eingesetzten Tools OPAL und ONYX werden von BPS entwickelt. Diese Werkzeuge haben für unseren Lehrstuhl aber auch für die TU Dresden und den Freistaat Sachen dementsprechend eine wichtige Rolle in der Lehre und bei Online-Prüfungen. Positiv anzumerken sind die zuverlässigen, schnellen und durchweg kompetenten Antworten bei Problemen, Fragen oder Anregungen. Obgleich einige der Anregungen (bspw. Kombination linearer und nicht-linearer Strukturen) aufgrund des programmiertechnischen und damit verbundenen finanziellen Aufwands leider auf eine Umsetzung warten lassen. Hier sollten die Wünsche und Anmerkungen zentral (hochschulweit oder landesweit) koordiniert gebündelt und priorisiert werden. Unser größter Wunsch hinsichtlich der Funktionalität von ONYX wäre es, eine Kombination von linearen und nichtlinearen ONYX-Tests zu realisieren.

\section{Rahmenbedingungen}

Wesentlich wäre es, die Rahmenbedingungen an der TU Dresden für Online-Prüfungen praxistauglich und konkret festzulegen, insbesondere im Spannungsfeld von Prüfungswertigkeit, Datenschutz, Identitätsprüfung, Prüfungsordnungen, aber auch hinsichtlich angepasster Weiterbildungsangebote für Lehrende und Lernende. Nach einem Jahr gibt es an der TU Dresden nach wie vor keine Lösung zum Thema datenschutzkonformer Identitätsprüfung bei Online-Prüfungen, was zwangsläufig zu einer Entwertung von Prüfungen hinsichtlich ihrer eigentlichen Aussagekraft über die Leistung eines Prüflings führt und den Wert der Bemühungen aller Beteiligten deutlich reduziert. Denkbar wäre es, ein eAssessment Center für Online-Prüfungen mit (mehreren) zentralen Räumlichkeiten zu schaffen.

\section{Zentrale Koordinierungsstelle}

Um ein lehrstuhlübergreifendes zukunftsfähiges, konkurrenzfähiges und attraktives Lehrangebot mit Online-Prüfungen anbieten zu können, ist eine dauerhafte personell, räumlich und technisch entsprechend ausgestattete 
zentrale Einrichtung an einer Hochschule erforderlich. Diese sollte nicht nur auf Zuruf verfügbar sein, sondern im Sinne einer exzellenten Hochschulentwicklung proaktiv die Lehrstühle ermutigen, neue Lehr- und Prüfungskonzepte umzusetzen.

In diesem Zusammenhang ist das "Zentrum für interdisziplinäre Lernen und Lehren“ ZiLL hervorzuheben, welches sich dieser Aufgabe an der TU Dresden angenommen hat und Kompetenzen, Informationen, Weiterbildungsangeboten sowie Ansprechpartner:innen für eine Umsetzung von Online-Angeboten bereitstellt. Hier ist zu hoffen, dass das ZiLL fester Bestandteil der Universitätsstruktur wird und damit langfristig erhalten bleibt.

Aber hervorzuheben sind auch das Engagement des Teams der TU-eigenen Messaging Plattform matrix.tu-dresden.de sowie die Task Force Digitale Prüfungen, bei der freiwillige Lehrende der Fakultät Maschinenwesen andere Lehrende bei Fragen zur Prüfungserstellung mit Ihren Erfahrungen beraten.

\section{eScouts}

eScouts sind ein hervorragendes Konzept und stellen für uns Keimzellen zur Wissensvermittlung zu und Umsetzung von Online-Inhalten dar. Interdisziplinäre eScout Teams und regelmäßige Treffen der eScouts könnten aus unserer Sicht dazu beitragen, das zugrunde liegende interdisziplinäre Potential wirklich auszuschöpfen. Zugleich wären längere Verträge für eScouts sinnvoll, um eine finanzielle Sicherheit zu geben und einen Knowhow-Transfer sicherzustellen. Dabei sollte überlegt werden, ob ein entsprechender finanzieller Anreiz für eScouts geschaffen wird, indem diese hinsichtlich Ihres Gehalts höher eingestuft werden als eine studentische Hilfskraft - immerhin sollten Weiterbildungen und Zusatzqualifikationen zur Befähigung als eScout erforderlich sein, die auch entsprechend honoriert werden müssten. Eine Absicherung der eScout Grundqualifikation bspw. über eine escout Summerschool oder ein eScout Zertifikat könnte das leisten und damit lukrative Studierendenjobs eröffnen.

\section{Fazit}

Unabhängig von den derzeitigen, pandemiebedingten Einschränkungen in der Präsenz- lehre sind Online-Prüfungen damit für die Autoren ein leistungsfähiges und zeitgemäßes Prüfungsformat.

\section{Anregungen}

Im Rahmen der turbulenten Semester sind eine Reihe von Ideen und Vorschlägen entstanden, mit denen wir anregen möchten bzw. deren Umsetzung aus unserer Sicht wünschenswert wäre und zu Effizienzsteigerungen führen könnte. Diese sollen im Folgenden zusammenfassend, stichpunktartig aufgelistet werden:

- Kombination linearer und nicht-linearer Navigation in ONYX-Tests

- Offline-Erstellung von ONYX-Tests

- fakultätsübergreifende Standards für Plattformen und Nomenklatur (MAXIMA)

- Einführungs-Webinare in OPAL, ONYX, MAXIMA für Studierende

- Server-Updates nur mit Ankündigung

- eine leistungsfähige OPAL-Instanz

- stochastischer Prüfungstestbot

- standardisierte eScout Qualifikation

- interdisziplinäre eScout Teams

- längerfristige eScout Beschäftigung

\section{Danksagung}

Wir danken Frau G. Haase, die uns im Sommersemester 2020 erfolgreich bei der Umsetzung der Online-Inhalte unterstützte und als eScout aus dem Projekt "Studiengänge flexibel gestalten" (SFG) des Medienzentrums der TU Dresden finanziert wurde, um zu einer Flexibilisierung der Studierbedingungen beizutragen.

\section{Literatur}

[1] https://www.bps-system.de/cms/products/opallearn-management/, 20.05.2021

[2] https://www.bps-system.de/cms/produkte/onyxtestsuite/, 20.05.2021

[3] https://bildungsportal.sachsen.de/portal/parentpage/institutionen/bps-bildungsportal-sachsengmbh/, 20.05.2021

[4] https://bildungsportal.sachsen.de/portal/parentpage/institutionen/arbeitskreis-e-learning-der-Irksachsen/, 20.05.2021

[5] https://doc.matrix.tu-dresden.de/, 20.05.2021

[6] https://tu-dresden.de/tu-dresden/organisation/rektorat/prorektor-bildung/zill, 20.05.2021 